\title{
LOCAL LINEAR EMBEDDING WITH MORPHABLE MODEL FOR FACE RECOGNITION
}

\author{
Xiaoming Bai, Baocai Yin, Qin Shi, Yanfeng Sun \\ Beijing Municipal Multimedia and Intelligent Software Key Lab, Beijing University of \\ Technology, Beijing 100022, China
}

\begin{abstract}
In this paper, we use local linear embedding and linear discriminant analysis for face recognition. Local linear embedding method is used to nonlinearly map high-dimensional face images to low-dimensional feature space. To recover space structure of face images, we use 3D morphable model to derive multiple images of a person from one single image. Experimental results on ORL and UMIST face database show that our method make impressive performance improvement compared with conventional Fisherface method.
\end{abstract}

Key words: Local linear embedding, Fisherface, Morphable model, Face recognition.

\section{INTRODUCTION}

PCA and LDA are two powerful tools utilized for data reduction and feature extraction in face recognition [1]. However face images are regarded as a nonlinear manifold in high-dimensional space [2], linear methods are bounds to ignore essential nonlinear structures that contains in manifold. Nonlinear reduction methods $[3,4]$ are presented to implement data reduction. LLE [2] performs with effective results. To recover structure of embedding manifold in low-dimensional space, a large number of training images taken from different viewpoints under different illumination conditions are needed to approximate distribution of manifold. These images are usually hardly available in real-world applications. Morphable model is proposed by Vetter [5]. Matching model to given facial images, 3D face 
could be reconstructed automatically. Reconstructed 3D face can be viewed from different viewpoints and under different illumination conditions.

Remainder of the paper is organized as follows: Section 2 explains LLE algorithm and its parameter selections. Section 3 describes method to derive multiple images from a single image with morphable model. Section 4 describes face recognition system based on LLE with morphable model. Section 5 presents experimental results. Section 6 gives conclusions.

\section{LLE ALGORITHM}

Main principle of LLE algorithm is to preserve local relation of data set in both embedding and intrinsic space [2]. LLE maps data set in highdimensional space globally to low-dimensional space. Dimensionality $d$ of intrinsic space, number of neighbors $k$ and regularization parameter $r$ are to be set [7].

To estimate $k$, we perform PCA on data set $X$ and get the $t$ largest eigenvalue $\lambda_{j}^{(i)}$, which satisfy $e \leq \sum_{j=1}^{t} \lambda_{j}^{(i)} / \sum_{j=1}^{D} \lambda_{j}^{(i)}$; Sample $x_{i}$ and its $t$ neighbor constructs a subspace $X_{i}$. PCA is performed on every $X_{i}$ to get the $l^{(i)}$ largest eigenvalue $\mu_{j}^{(i)}$, which satisfy $e^{\prime} \leq \sum_{j=1}^{(i)} u_{j}^{(i)} / \sum_{j=1}^{i} u_{j}^{(i)}$; Lastly, we estimate $k=\max _{i=1 \ldots n}\left\{l^{(i)}\right\}$. To estimate local dimensionality, we perform PCA on each $k$-neighbor of sample $x_{i}$ to get the $p^{(i)}$ largest eigenvalues $\gamma_{j}^{(i)}$, which satisfy $e^{\prime \prime} \leq \sum_{j=1}^{j^{(i)}} \gamma_{j}^{(i)} / \sum_{j=1}^{k} \gamma_{j}^{(i)}$. Then we compute mean value of the $p^{(i)}$ and let $d=\frac{1}{n} \sum_{i=1}^{n} p^{(i)}$. For computation of regularization parameter $r$, we use same method as $d$. For each $k$-neighbor of sample $x_{i}, \sigma_{i}^{2}=\frac{1}{k-d} \sum_{j=d+1}^{k} \gamma_{j}^{(i)}$ and $r=\frac{1}{n} \sum_{i=1}^{n} \sigma_{i}^{2}$.

\section{DERIVING MULTIPLE IMAGES FROM A SINGLE IMAGE}

Basic theory of morphable model is linear combination of object class [6]. Given an input facial image, through model matching process, 3D face 
model of specific person can be reconstructed. New illumination model can be applied to 3D face model to generate multiple images under different lighting conditions [6]. Figure 1 illustrates some example images derived from a single image. Left column is a single image of a person. Right columns are multiple images derived from the single image by rotating and adding new lighting to the $3 \mathrm{D}$ face model.

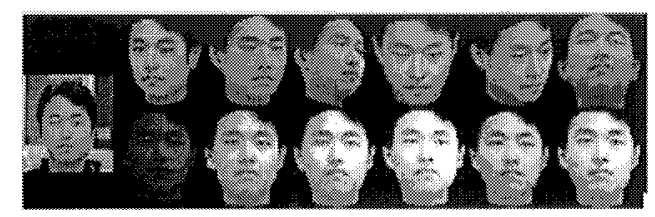

Figure. I Multiple images derived from a single image

\section{LLE BASED FACE RECOGNITION}

LLE algorithm needs samples cover full manifold and FLD method can not be applied to face recognition problems where only one training image per person is available. Multiple training images per person are needed to compute within-class scatter matrix. Morphable model is applied into face recognition system to derive multiple samples from a single example image. Framework of our face recognition system is as shown in figure 2. The part above broken line is to derive multiple images from a single image. All images derived from the single image consist into the training sample set.

\section{EXPERIMENTAL RESULTS}

To verify effectiveness of the proposed approach, we also develop Fisherface method as benchmark according to [8]. Performance of these approaches are evaluated and compared using the Recognition Rate (RR) standard definded as $R(j)=\frac{N_{j}}{N}$. Where $N$ is total number of test sample set, $N_{j}$ is number of test images recognized correctly in the top $j$ candidate.

Experiments are conducted on ORL and UMIST database. ORL database [9] contains 40 distinct persons with ten images per person. Images are taken at different time instance, with varying lighting conditions, facial expressions and poses. In our experiments, the approximately frontal examples with neutral expression of each subject in database are chosen as 
example images to form training set. 20 novel face images derived from example image of each subject using method described in section 3 are utilized as training sample images for each person. Performance curves of our method and benchmarks are shown in figure 3. The rank-1(first-choice) recognition rate of Fisherface method is $75.1 \%$, while our proposed method is $83.7 \%$. $\left(k=37 d=57 r=1.3 \times 10^{-4}\right)$

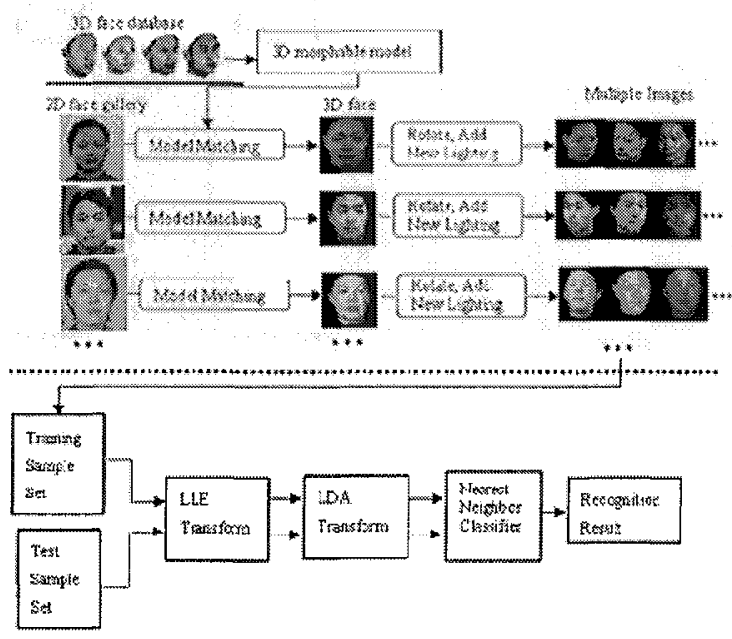

Figure. 2. Framework of face recognition system

UMIST database [10] is a multi-view database, consisting of 575 images of 20 people, each covering a wide range of poses from profile to frontal views. For each subject, one normal face is chosen as example image to form training set. 45 novel face images derived from example image of each subject using method described in section 3 are utilized as training sample images for each person. Performance curves of our method and benchmark are shown in figure 3. The rank-1 recognition rate of our method is $88.7 \%$, Fisherface method is $80.6 \%$. $\left(k=45 d=23 r=1.2 \times 10^{-4}\right)$

\section{CONCLUSIONS}

In this paper, LLE and LDA are combined for face recognition. Lowdimensional manifold structure of face space is obtained by LLE. LDA is used to implement classification on description space. 3D morphable model is utilized to derive multiple images from a single example image to form training set for LLE+LDA. Experiments on ORL and UMIST face database demonstrate impressive performance of our method. 

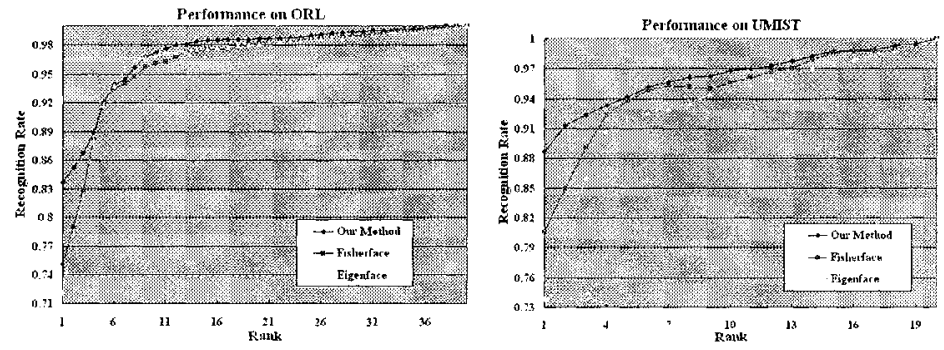

Figure. 3 Performance Comparisons on ORL and UMIST Database

\section{ACKNOWLEDGEMENTS}

The authors would like to thank AT\&T Laboratories Cambridge for providing the ORL face database and Dr. D. Graham and Dr. N. Allinson for providing the UMIST face database. This work is supported by the Beijing Educational Committee Project of China (2002KJ001, KM200310005025), the National Natural Science Project of China (60375007).

\section{REFERENCES}

[1] R. Chellapa, C. Wilson, S. Sirohey, Human and machine recognition of faces: a survey, Proceedings of the IEEE, 1995, 83(5):705-741.

[2] Sam. T. Roweis, L. K. Saul, Nonlinear Dimensionality Reduction by Locally Linear Embedding, Science, Vol 290, 2000: 2323-2326.

[3] T. Hastie, Principal curves and surfaces, Laboratory for computational Statistics Stanford University, Dept. of Statistics Technical Report 11, 1984.

[4] J. B. Tenenbaum, de Silva, V. \& Langford, J. C, A global geometric framework for nonlinear dimensionality reduction, Science, Vol 290, 2000: 2319-2323.

[5] Blanz V, Vetter T. A morphable model for the synthesis of 3D faces. In Proceeding of SIGGRAPH'99, Los Angeles: ACM Press, 1999: 187-194.

[6] Yongli $\mathrm{Hu}$, et al, An Improved Morphable Model for 3D Face Synthesis, International Conference on Machine Learning and Cybernetics, Vol. 6, 2004: 4362-4367.

[7]De Ridder, et al, Locally linear embedding for classification, Technical report PH-2002-01, Dept. of Imaging Science \& Technology, Delft University of Technology, 2002: 1-15.

[8] P. N. Belhumeur, et al, Eigenfaces vs. Fisherfaces: Recognition using class specific linear projection, IEEE Trans. Pattern Anal.Machine Intell, Vol. 19, May 1997: 711-720.

[9] ORL face database. AT\&T Laboratories, Cambridge, U. K. [Online]. Available: http://www.cam-orl.co.uk/facedatabase.html

[10] D. B. Graham and N. M. Allinson, Characterizing virtual eigensignatures for general purpose face recognition, in Face Recognition: From Theory to Applications, H. Wechsler, P. J. Phillips, V. Bruce, F. Fogelman-Soulie, and T. S. Huang, Eds., 1998, vol. 163, NATO ASI Series F, Computer and Systems Sciences, pp. 446-456. 